\title{
On Bayesian procedure for maximum earthquake magnitude estimation
}

\author{
Andrzej Kijko \\ Aon Benfield Natural Hazard Centre, University of Pretoria, Republic of South Africa
}

\begin{abstract}
This work is focused on the Bayesian procedure for the estimation of the regional maximum possible earthquake magnitude $m_{\max }$. The paper briefly discusses the currently used Bayesian procedure for $m_{\max }$, as developed by Cornell, and a statistically justifiable alternative approach is suggested. The fundamental problem in the application of the current Bayesian formalism for $m_{\max }$ estimation is that one of the components of the posterior distribution is the sample likelihood function, for which the range of observations (earthquake magnitudes) depends on the unknown parameter $m_{\max }$. This dependence violates the property of regularity of the maximum likelihood function. The resulting likelihood function, therefore, reaches its maximum at the maximum observed earthquake magnitude $m_{\max }^{o b s}$ and not at the required maximum possible magnitude $m_{\max }$. Since the sample likelihood function is a key component of the posterior distribution, the posterior estimate of $\hat{m}_{\max }$, is biased. The degree of the bias and its sign depend on the applied Bayesian estimator, the quantity of information provided by the prior distribution, and the sample likelihood function. It has been shown that if the maximum posterior estimate is used, the bias is negative and the resulting underestimation of $m_{\max }$ can be as big as 0.5 units of magnitude. This study explores only the maximum posterior estimate of $m_{\max }$, which is conceptionally close to the classic maximum likelihood estimation. However, conclusions
\end{abstract}

Correspondence: Andrzej Kijko, Aon Benfield Natural Hazard Centre, Room 4-30, Mineral Sciences Building, University of Pretoria, Pretoria 0002 Republic of South Africa.

Tel. +27.12.420.3613 - Fax: +27.12.362.5219.

E-mail: andrzej.kijko@up.ac.za

Key words: maximum earthquake magnitude, Bayesian procedure.

Acknowledgments: the author wishes to acknowledge and thank Dr DH Weichert for his constructive contribution to the final version of this manuscript which was provided during his visit to South Africa. Ms Ansie Smit is thanked for her careful reading of the manuscript, critical review, suggestions and very helpful comments.

Received for publication: 1 January 2012.

Revision received: 23 April 2012.

Accepted for publication: 26 April 2012.

This work is licensed under a Creative Commons Attribution NonCommercial 3.0 License (CC BY-NC 3.0).

(C) Copyright A. Kijko, 2012

Licensee PAGEPress, Italy

Research in Geophysics 2012; 2:e7

doi:10.4081/rg.2012.e7 regarding the shortfall of the current Bayesian procedure are applicable to all Bayesian estimators, e.g. posterior mean and posterior median. A simple, ad hoc solution of this non-regular maximum likelihood problem is also presented.

\section{Introduction}

Many people recall 11 March 2011, the day on which the massive 9.0 $\left(M_{\mathrm{W}}\right)$ magnitude earthquake and tsunami struck the north-eastern coast of Japan, killing more than 30,000 people. As a result, three reactors at the Fukushima Daiichi nuclear facility overheated, causing meltdowns that released large amounts of radioactive material into the air. The enormous size of the earthquake took most people by surprise. This earthquake, known as the Tohoku earthquake, overshadowed a similar event that took place on 17 July 2007. On that day, an earthquake of magnitude $M_{\mathrm{J}} 6.8$ hit Kashiwazaki-Kariwa, the world's largest nuclear power plant complex, causing radioactive leaks from two reactors and a fire at Reactor No. 3. The reason given for the damage to the plant, as indicated in a series of reports issued by a team of international experts led by the International Atomic Energy Agency (IAEA) was that Levels of seismic ground motion estimated in the design process were very significantly exceeded by the event.... ${ }^{1}$ Most Japanese nuclear plants are built to withstand earthquakes of magnitude $M_{\mathrm{J}}=6.5$, while the earthquake of 17 July 2007 had a magnitude of 6.8 .

The nuclear safety breaches at the Fukushima Daiichi and the Kashiwazaki-Kariwa nuclear power facilities serve as pertinent reminders of the importance of rigorous seismic-hazard assessment of critical-structure sites. This is especially relevant to the correct assessment of the regional-characteristic, maximum possible earthquake magnitude $m_{\max }$.

In his contribution to the NP-4726 EPRI report, Cornell ${ }^{2}$ presents a Bayesian procedure, referred to hereafter as Cornell's Bayesian Procedure (CBP) for the assessment of the regional-characteristic, maximum possible earthquake magnitude $m_{\max }$. The CBP was presented as a possible solution in the broad context of problems associated with the determination of $m_{\max }$. But Cornell ${ }^{2}$ does not explicitly confirm the type of his Bayesian estimator. This paper aims to provide a discussion of the simplest (the maximum posterior estimate) of the CBP and its potential shortfalls.

The essence of the current CBP for the estimation of $m_{\max }$ consists in combining the following two sources of information:

i. prior knowledge of the distribution of the maximum possible earthquake magnitude $m_{\max }, \pi\left(m_{\max }\right)$

and

ii. likelihood function $L\left(\mathrm{~m} \mid m_{\max }\right)$ of a random sample of earthquake magnitudes $\mathbf{m}=\left(\mathrm{m}_{1}, \mathrm{~m}_{2}, \ldots, \mathrm{m}_{\mathrm{n}}\right)$.

The knowledge of a prior distribution $\pi\left(m_{\max }\right)$ comes from the worldwide database of $m_{\max }$ for different seismogenic regions. Cornell ${ }^{2}$ describes in detail how the prior $\pi\left(m_{\max }\right)$ had been established. All 
seismic active regions were grouped into different categories (domains) according to crustal type, intra- or interplate, source origin, age, tectonic stresses, tectonic history, etc. For more details see the studies of Coppersmith ${ }^{3}$ and Johnston. ${ }^{4}$

In this short paper, it is assumed that the prior information on $m_{\max }$ is available and correct, that it was established independently on the sample of earthquake magnitudes $m=\left(m_{1}, \ldots, m_{n}\right)$, and that it can be expressed in terms of the probability density function $\pi\left(m_{\max }\right)$. In statistics, $\pi\left(m_{\max }\right)$ is known as a prior distribution, which describes current knowledge about the unknown parameter $\left(m_{\max }\right)$, prior to taking a random sample of observations; in this case, earthquake magnitudes m.

The second source of information on $m_{\max }$ is the sample likelihood function $L\left(\mathrm{~m} \mid m_{\max }\right)$ of $n$ independent earthquake magnitudes $m_{\mathrm{i}}$.

$$
L\left(\mathbf{m} \mid m_{\max }\right)=\prod_{i=1}^{n} f_{M}\left(m_{\mathrm{i}} \mid m_{\max }\right)
$$

where $f_{M}\left(\mathrm{~m} \mid m_{\max }\right)$ denotes the probability density function (PDF) of the earthquake magnitude m. Following Cornell, ${ }^{2}$ it is assumed that earthquake magnitudes mi are independent random values, distributed according to the classic frequency-magnitude Gutenberg-Richter relation and bounded from the top by the maximum possible earthquake magnitude $m_{\max }$. It is assumed that all events having a magnitude greater than or equal to $m_{\min }$ are recorded. Magnitude $m_{\min }$ is the known level of completeness. For the Gutenberg-Richter relation, the respective PDF and cumulative distribution function (CDF) of earthquake magnitude are continuous and equal to $0^{5,6}$ :

$$
f_{M}\left(m \mid m_{\max }\right)= \begin{cases}0 & \text { for } m_{\min }<m \\ \frac{\beta \exp \left[-\beta\left(m-m_{\min }\right)\right]}{1-\exp \left[-\beta\left(m_{\max }-m_{\min }\right)\right]} & \text { for } m_{\min } \leq m \leq m_{\max }(2) \\ 0 & \text { for } m>m_{\max }\end{cases}
$$

and

$$
F_{M}\left(m \mid m_{\max }\right)= \begin{cases}0 & \text { for } m<m_{\min } \\ \frac{1-\exp \left[-\beta\left(m-m_{\min }\right)\right]}{1-\exp \left[-\beta\left(m_{\max }-m_{\min }\right)\right]} & \text { for } m_{\min } \leq m \leq m_{\max } \\ 1 & \text { for } m>m_{\max }\end{cases}
$$

where $\beta=b \ln (10)$, and $b$ is the parameter of the frequency-magnitude Gutenberg-Richter relation.

The distribution which summarizes both the prior knowledge about $m_{\max }$ and the knowledge coming from the observed magnitudes $\mathbf{m}$ is known as the posterior distribution of $m_{\max }$ and takes the form

$$
f_{m_{\max }}\left(m_{\max } \mid \mathbf{m}\right)=\frac{\pi\left(m_{\max }\right) L\left(\mathbf{m} \mid m_{\max }\right)}{\int \pi\left(m_{\max }\right) L\left(\mathbf{m} \mid m_{\max }\right) \mathrm{d} m_{\max }}
$$

The posterior density distribution (4) replaces the likelihood function $L\left(\mathbf{m} \mid m_{\max }\right)$ as an expression that incorporates all available information on parameter $m_{\max }$. There are at least three Bayesian analogs of the maximum likelihood point estimators. The first is the maximum $a$ posterior estimate, known as the MAP estimate. The MAP estimate corresponds to the magnitude at which the posterior distribution (4) achieves its maximum. The second Bayesian estimator is the posterior mean, determined as the first moment (the mean value) of the posterior distribution (4). The third Bayesian estimator is the posterior median. ${ }^{7}$ This study explores only the MAP estimate of $m_{\max }$ which is close to the classic maximum likelihood procedure; however, all conclusions regarding the shortfall of the current CBP are by default applicable to all three Bayesian estimators. Therefore, and for the following definition of the MAP estimate, the value of $m_{\max }$, which maximises the posterior distribution $f m_{\max }\left(m_{\max } \mid \mathbf{m}\right)$ will be analyzed.

\section{Why is the current procedure for $m_{\max }$ evalua- tion flawed?}

The fundamental problem with the current CBP for $m_{\max }$ evaluation lies in the properties of the sample likelihood function $L\left(\mathrm{~m} \mid m_{\max }\right)$. The sample likelihood function is expected to be of such a form that the probability to observe magnitudes $\mathbf{m}$ reaches its maximum when $m_{\max }$ is equal to the true $m_{\max }{ }^{7}$ Unfortunately, the likelihood function (1) does not fulfil this condition. If, in the construction of the likelihood function the PDF of the Gutenberg-Richter is used, (formula 2), the likelihood function reaches its maximum at the maximum observed magnitude $m_{\max }^{o b s}$ and not, as would be expected, at the maximum possible magnitude $m_{\max }$. This fact is illustrated in Figure 1 where the values of $m_{\max }$ that are smaller than $m_{\max }^{o b s}$ are not allowed; however, values larger than $m_{\max }^{o b s}$ would give a smaller likelihood.

Since by default $m_{\max }^{o b s} \leq m_{\max }$ the sample likelihood function (1) provides an estimate of $\mathrm{m}_{\max }$, (denoted as $\hat{m}_{\max }$ ) which is systematically underestimated. This fact is well known in statistics ${ }^{7,8}$ and it takes place when the range of observations depends on an unknown parameter. Such dependence violates the property of regularity of the maximum likelihood function. ${ }^{9}$

When the likelihood function is non-regular, it achieves its maxi-

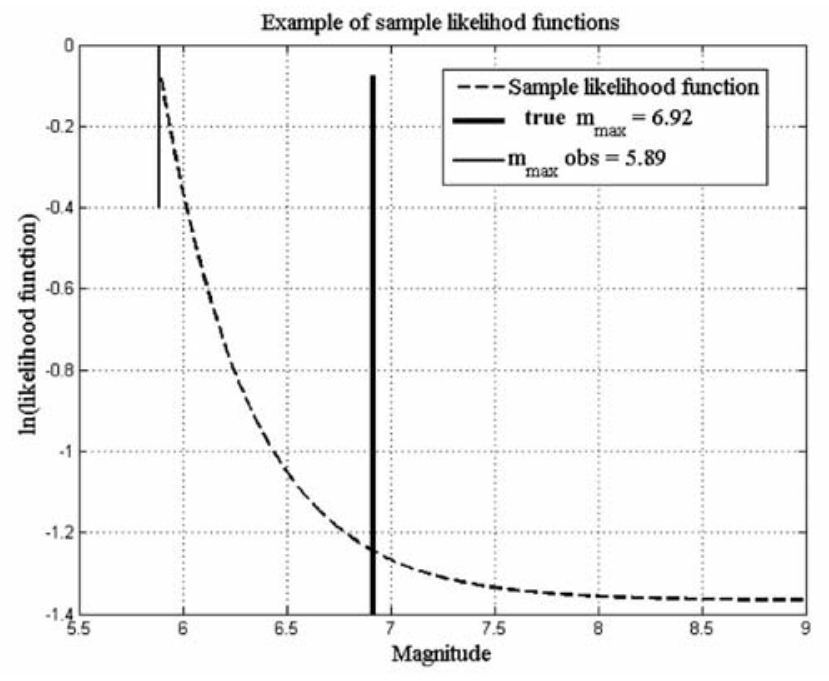

Figure 1. Illustration of the sample likelihood function $L\left(\mathrm{~m} \mid \mathrm{m}_{\max }\right)$. Since the range of the observed magnitudes depends on the unknown $m_{\max }$ the function reaches its maximum at the maximum observed magnitude $m_{\max }^{o b s}$ and not, as would be expected, at the maximum possible, $\mathrm{m}_{\max }$. 
mum at the maximum observed value, in this case $\operatorname{MAX}(\mathrm{m})=m_{\max }^{\text {obs }} ; \mathrm{a}$ result that is too small. In the CERN classic textbook of statistics, ${ }^{10}$ such an instance is referred to as an academic example of a poor maximum likelihood estimate. A solution to this poor behavior of the sample likelihood function, which includes a description of the phenomenon from point of view of theory of estimation is provided, for example, by Bain and Engelhardt. ${ }^{11}$

Given that the sample likelihood function is part of the posterior distribution (4), the MAP estimation procedure has the same flaw: its estimate of $m_{\max }$ is systematically underestimated. The degree of underestimation depends on the quantity of information provided by each of the two components of the posterior distribution, namely the prior distribution $\pi\left(m_{\max }\right)$ and sample likelihood function $\mathrm{L}\left(\mathrm{m} \mid m_{\max }\right)$. The

underestimation of $\hat{m}_{\max }$ is minimal when the prior information is well defined, accurate and precise, and $\mathrm{L}\left(\mathrm{m} \mid m_{\max }\right)$ is based on a short catalog with only a few weak events. In such an instance, all the information in $m_{\max }$ will come from the prior distribution. However, in an instance where the sample likelihood function contains a significant amount of information (e.g. it is based on a long span of catalog containing many events, including historic earthquakes), the posterior distribution (4) will be dominated by $\mathrm{L}\left(\mathrm{m} \mid m_{\max }\right)$. Then maximum of (4)

will be between $m_{\max }^{o b s}$ and the true $m_{\max }$. Since $m_{\max }^{o b s} \leq m_{\max }$ this instance in most cases will lead to the biased, underestimated value of $\hat{m}_{\max }$.

Illustrations of such underestimations, obtained through MonteCarlo simulations, and applied for the two different prior distributions as in Figure 2, are shown in Figures 3 and 4. The first prior distribution is based on work by Petersen et al. ${ }^{12}$ The second prior distribution is the classic, double truncated Gaussian PDF. ${ }^{3}$ In both instances, the sample likelihood function is derived from a random sample of earthquake magnitudes, generated according to the Gutenberg-Richter magnitude distributions (2), where $m_{\min }=4.0, m_{\max }=6.92, \beta=2.0$, the mean activity rate $\lambda=10$ (events/year), and time span of the catalog is equal to 100 years. It is important to note that all quantitative assessments resulting from the Monte-Carlo simulation depends on the difference between $m_{\max }$ and $m_{\min }$. The smaller the difference, the fewer the number of earthquakes needed to obtain the same effect. In the discussed case of $m_{\min }=4.0$ and $m_{\max }=6.92$, the difference $m_{\max }-m_{\min }$ is approximately 3 . A reduction of this difference by 1 unit of magnitude leads to a reduction in the number of earthquakes by approximately one order, which means that the same assessment of $m_{\max }$ can be reached with 10 times less earthquakes if the level of completeness is $m_{\max }=5.0$.

Figure 3 shows the results of the Monte-Carlo simulations of the $m_{\max }$ MAP assessment by the current CBP, when Petersen's distribution is used as the prior distribution. The mean value of Peterson's $m_{\max }$ distribution is 6.92 (standard deviation 0.32 ). Results of similar simulations for the Gaussian prior distribution are shown in Figure 4. The applied Gaussian distribution is truncated from both sides; from the left at $m_{\max }^{o b s}$ and from the right at the magnitude 9.5. The mean value of the distribution (before truncation) is the same as for the Petersen's distribution, i.e. 6.92 (standard deviation 0.32).

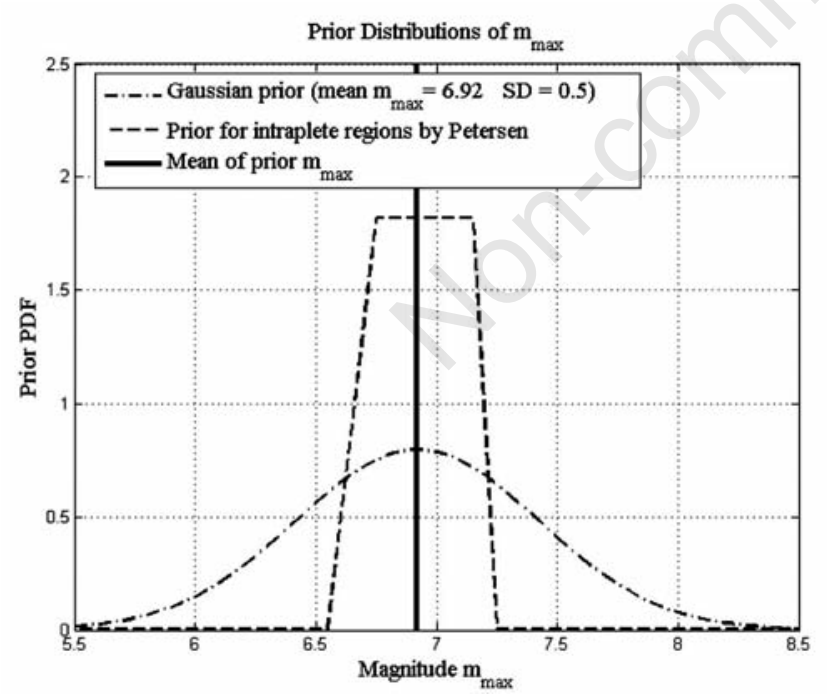

Figure 2. The two prior distributions of the maximum possible earthquake magnitude, $m_{\max }$, used in this study. The first (dashed line) illustrates the distribution of $m_{\max }$ for intraplate regions. The mean value of the prior distribution is equal to 6.92 and its standard deviation is $\mathbf{0 . 3 2}$. The second applied prior distribution (dash-dot line) is Gaussian, truncated from both sides. From the left it is truncated at the magnitude $m_{\max }^{o b s}$ and from the right at magnitude 9.5. The mean value of the distribution (before truncation) and its standard deviation are the same as for the distribution by Petersen $e t$ al. ${ }^{12}$

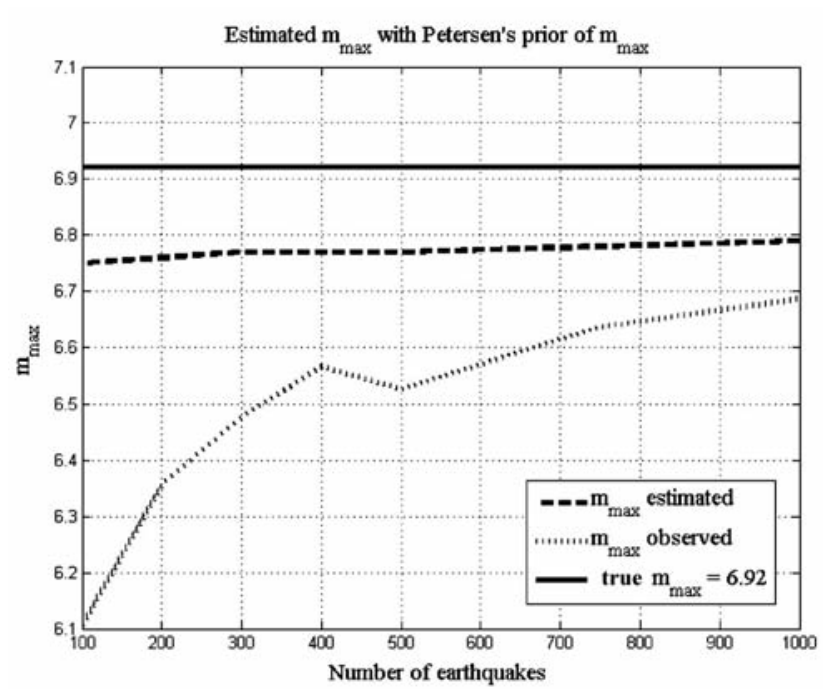

Figure 3. The $m_{\max }$ estimation using the current Cornell's Bayesian Procedure with the prior based on work by Petersen $e t$ $a l .{ }^{12}$ The maximum of the posterior distribution is between $m_{\max }^{o b s}$ and true $m_{\max }$. In the above example, earthquake magnitudes were generated according to the Gutenberg-Richter magnitude distributions (2), where $\operatorname{mmin}=4.0, m_{\max }=6.92$, and $\beta=2.0$. The results depend on the assumed difference between $m_{\max }$ and $m_{\min }$. A reduction of this difference by 1 unit of magnitude leads to a reduction in the number of earthquakes by approximately one order, which means that the same effect can be reached with the help of 10 times less earthquakes if the assumed level of completeness is $m_{\min }=5.0$. 


\section{Correction of the procedure}

Correction of the current CBP for the maximum earthquake magnitude estimation can be made in several ways. Essentially, two approaches can be applied. The first is a palliative approach in which the effect of the bias in the maximum likelihood $m_{\max }$ estimation is assessed and removed. The second approach is where the cause of the bias is removed. A comprehensive discussion of the two approaches will be the subject of a separate study. In this short paper, only the simplest case of the palliative correction procedure is presented.

The reasoning leading to the correction of the CBP can be summarized as follows: if the unbiased maximum likelihood estimator of $m_{\max }$ is known, then the current Bayesian estimator of $m_{\max }$ would also produce an unbiased $m_{\max }$. If so, the correction of the CBP can be made as follows: first, the bias of the maximum likelihood estimation of $m_{\max }$ must be assessed. Then, in formula (4), the sample likelihood function $L\left(\mathrm{~m} \mid m_{\max }\right)$ is replaced with the same likelihood function, but shifted by the amount of estimated bias. This can be modified relatively easily since the formulas describing the bias of maximum likelihood estimation of $m_{\max }$ are known. It is interesting to note that the suggested solution, which essentially consists of the replacement of an unknown parameter by its unbiased estimate, is not new. In statistical literature it is known as the Expectation-Maximisation (EM) algorithm and uses the principle of the maximum likelihood to obtain unbiased estimates of missing data. ${ }^{13}$

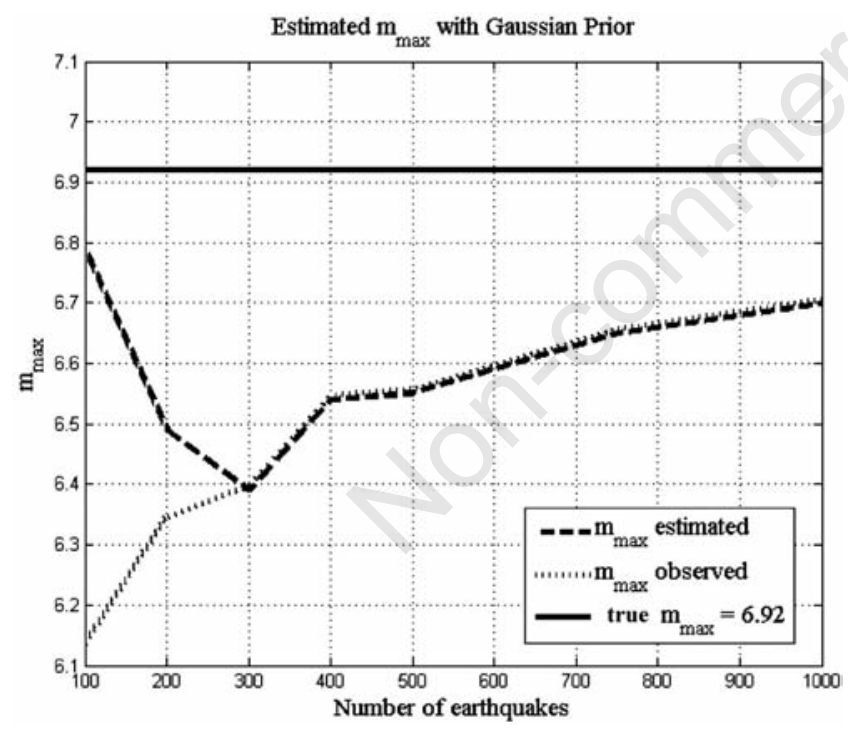

Figure 4. The $m_{\max }$ estimation using the current Cornell's Bayesian Procedure. The Gaussian prior distribution is truncated from both sides; from the left at $m_{\max }^{o b s}$ and from the right at magnitude 9.5. The mean of the distribution (before truncation) is $\mathbf{6 . 9 2}$ (standard deviation 0.32). As the number of events increases, the posterior distribution is dominated by the contribution of the sample likelihood function with a maximum at magnitude. $m_{\max }^{o b s}$ The earthquake magnitudes were generated according to the Gutenberg-Richter magnitude distributions (2), where $m_{\min }=4.0$, $m_{\max }=6.92$, and $\beta=2.0$. Above results depend on assumed difference between $m_{\max }$ and $m_{\min }$. A reduction of this difference by 1 unit of magnitude leads to a reduction of the number of earthquakes approximately by one order. It means that the same effect can be reached with the help of 10 times less earthquakes, if the assumed level of completeness is $m_{\text {min }}=5.0$.
At least two formulas describing the bias in $m_{\max }$ estimation are currently in use. The simpler formula, derived by Tate, ${ }^{14}$ when applied to estimation of $m_{\max }$, takes the form: ${ }^{15,16}$

$$
\Delta=\frac{1}{n f_{M}\left(m_{\max }^{o b s} \mid m_{\max }\right)}
$$

In the above formula, $\Delta$ denotes bias in $m_{\max }$ estimation and $n$ is number of seismic events with magnitudes equal to or exceeding the level of completeness $m_{\max }$. If Tate's formula is applied to the frequency-magnitude Gutenberg-Richter relation with PDF (2), the bias in the $m_{\max }$ estimation takes the form: ${ }^{15,16}$

$$
\Delta=\frac{1-\exp \left[-\beta\left(m_{\max }-m_{\min }\right)\right]}{n \exp \left[-\beta\left(m_{\max }^{\text {obs }}-m_{\min }\right)\right]}
$$

The second formula describing the bias in $m_{\max }$ estimation is:

$$
\Delta=\int_{m_{\min }}^{m_{\max }}\left[F_{M}\left(m_{\max }^{o b s} \mid m_{\max }\right)\right]^{n} d m
$$

where $F_{M}\left(m_{\max }^{o \text { obs }} \mid m_{\max }\right)$ denotes the CDF of earthquake magnitude.

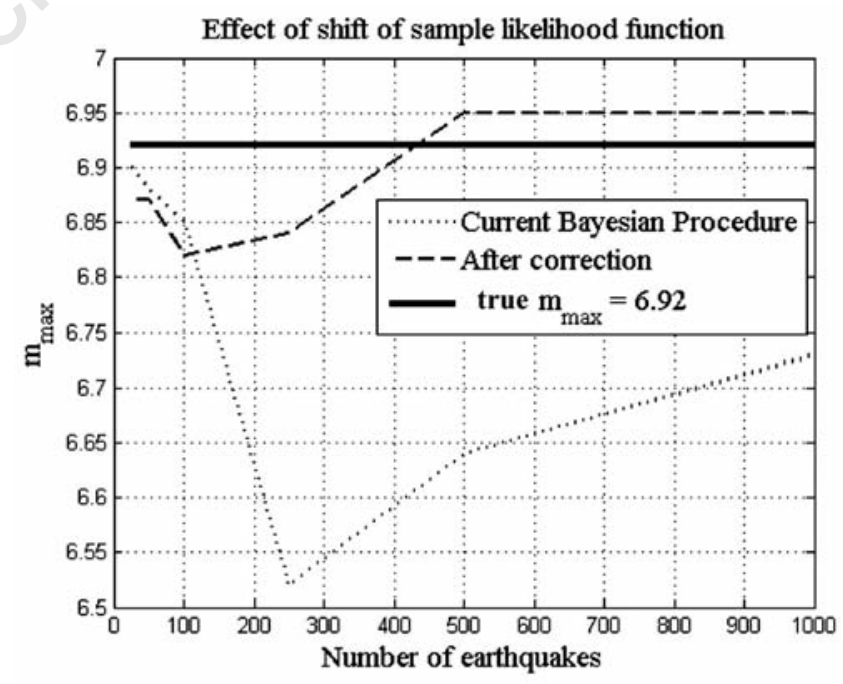

Figure 5. Comparison of performances of the $m_{\max }$ estimation using the current and corrected Cornell's Bayesian Procedures (CBPs). For a small number of events, (less than ca. 100), both the current and the corrected CBP perform similarly. In both cases, the sample likelihood function is weak and most of the information comes from the prior distribution. As the number of events increases, the modified CBP provides a better assessment of $m_{\max }$ than the original one. The synthetic earthquake magnitudes were generated according to the Gutenberg-Richter magnitude distributions (2), where $m_{\min }=4.0, m_{\max }=6.92$, and $\beta=2.0$. The results depend on the assumed difference between $m_{\max }$ and $m_{\min }$. A reduction of $m_{\max }-m_{\min }$ by 1 unit of magnitude leads to a reduction of the number 100 to approximately 10 , which means that the same effect can be reached with only 10 earthquakes if the level of completeness is $m_{\min }=5.0$. 
Cooke ${ }^{17}$ was the first to derive equation (7). It is interesting to note the difference between equation (7) and the original equation derived by Cooke. ${ }^{17}$ The former provides an equation in which the upper limit of integration is $m_{\max }^{o b s}$. In equation (7) the upper limit of integration is the unknown $m_{\max }$. An obvious effect of this difference between the two equations is that the relation in equation (7) is more difficult to implement; however, it provides a more accurate estimate of bias when the number of observations is small.

For the frequency-magnitude Gutenberg-Richter relation, with the CDF (3), equation (7) takes the form: ${ }^{18}$

$$
\Delta=\frac{E_{1}\left(n_{2}\right)-E_{1}\left(n_{1}\right)}{\beta \exp \left(-n_{2}\right)}+m_{\min } \exp (-n)
$$

where $n_{1}=n /\left\{1-\exp \left[-\beta\left(m_{\max }-m_{\min }\right)\right]\right\}, n_{2}=n_{1} \exp \left[-\beta\left(m_{\max }-m_{\min }\right)\right]$, and $E_{1}(\cdot)$ denotes an exponential integral function.

Since solution (8), based on the equation by Cooke, ${ }^{17}$ provides an estimate of the bias with a significantly lower mean squared error than the respective equation based on the equation (6) by Tate, ${ }^{14,18}$ formula (8) has been used in all further calculations.

The effect of implementing the above correction of the CBP (in case of Gaussian prior) is shown in Figure 5. This figure shows that for a small number of events (less than approx. 100 events with magnitude $\geq 4.0$ ), both the current and corrected CBP perform similarly. It should be remembered that this number of events depends on the difference between $m_{\max }$ and $m_{\max }$. The smaller the difference, the fewer the number of earthquakes needed to obtain the same assessment of $m_{\max }$.

This is the case when the sample likelihood function is weak and most of the information on $m_{\max }$ is determined by the prior distribution. As the number of events increases, so does the difference in the performance of both procedures. With the increase in the number of earthquakes, the assessment of the bias (8) becomes increasingly more accurate, the maximum of the shifted sample likelihood function becomes close to the true $m_{\max }$ and, consequently, the modified CBP procedure provides a better assessment of $m_{\max }$ than the current one. The difference in performance of the two procedures decreases only for a very large number of events when the magnitude of the maximum observed event $m_{\max }^{\text {obs }}$ approaches $m_{\max }$.

It is important to note that Cornell, the author of the discussed Bayesian procedure, ${ }^{2}$ was perfectly aware of all the limitations of his approach. In fact, he suggests not using the procedure at all. ${ }^{2}$ In the instance where the sample likelihood function dominates the posterior distribution, (e.g. this instance is based on a catalog containing many events, including historic earthquakes), he suggests replacing the Bayesian formalism by the bias reduction practice similar to those of Pisarenko ${ }^{15}$ and Kijko. ${ }^{18}$ For this purpose, he proposes to apply the fiducial distribution of $m_{\max }{ }^{19}$ In contrast, when the sample likelihood function is weak (the number of events is small and/or the difference between $m_{\max }$ and $m_{\min }$ is large), he stated that the current Bayesian formalism cannot provide a reliable estimate of $m_{\max }$.

\section{Conclusions}

It has been shown that the currently used Bayesian procedure for the estimation of the regional maximum possible earthquake magnitude has a mathematical flaw which leads to bias of the estimated value of $m_{\max }$. The cause of the bias is one of the components of the posterior distribution being the sample likelihood function for which the range of observations (earthquake magnitudes) depends on the unknown parameter $m_{\max }$.This dependence violates the rule of optimal properties of maximum likelihood estimators, known as the condition of regularity. ${ }^{9}$ If the condition of regularity is not fulfilled, the resulting likelihood function reaches its maximum at the maximum observed earthquake magnitude $m_{\max }^{o b s}$ and not at the required maximum possible magnitude $m_{\max }$.

Since the sample likelihood function constitutes a key component of the posterior distribution, the posterior estimate of $m_{\max }$, (denoted as $\hat{m}_{\text {max }}$ ) is biased. The degree of the bias and its sign depend on the applied Bayesian estimator, the prior distribution, and the number and magnitudes of seismic events used to construct the sample likelihood function.

The Monte-Carlo simulations show that if the MAP Bayesian estimator is used, the bias is negative and the underestimation of $m_{\max }$ by the current CBP can reach 0.5 units of magnitude. The degree of underestimation depends on the amount of information provided by each of two components to the posterior distribution: namely, the prior distribution $\pi\left(m_{\max }\right)$, and the sample likelihood function $\mathrm{L}\left(\mathrm{m} / m_{\max }\right)$. If the prior information is well defined and $\mathrm{L}\left(\mathrm{m} \mid m_{\max }\right)$ is based, for example, on a short instrumental catalog with only a few events, the underestimation of $\hat{m}_{\max }$ will be minimal since all the information on $m_{\max }$ is provided by the prior distribution. In the opposite case, when the sample likelihood function is derived from many events, including historic earthquakes, the posterior distribution is dominated by $\mathrm{L}\left(\mathrm{m} \mid m_{\max }\right)$ and its maximum is between $m_{\max }^{o b s}$ and the true $m_{\max }$. Since $m_{\max }^{o b s} \leq m_{\max }$ this instance leads to the underestimation of $m_{\max }$.

The study explores only the MAP estimate of $m_{\max }$ which is conceptionally close to the classic maximum likelihood procedure. However, conclusions regarding the shortfall of the CBP are applicable to all Bayesian estimators which utilize the sample likelihood function derived according to formula (1). For example, the second Bayesian estimator, the posterior mean, in contrast to MAP, overestimates the value of $m_{\max }$. The overestimation can reach a value of one unit of magnitude. Further studies are underway and a generic solution for the problem is being developed. These will be the subject of future reports.

\section{References}

1. International Atomic Energy Agency (IAEA). Third IAEA Report on Kashiwazaki-Kariwa nuclear power plant, 2009. Available from: http://www.iaea.org/newscenter/news/2009/ kashiwazaki290109. html

2. Cornell CA. Statistical analysis of maximum magnitudes in the earthquakes of stable continental regions. In: J Schneider (ed). Seismic hazard methodology for the Central and Eastern United States. The earthquakes of stable continental regions. Vol. 1. Assessment of large earthquake potential. Palo Alto, CA, USA: EPRI;1994. NP-4726, pp 5-27.

3. Coppersmith KJ. Conclusions regarding maximum earthquake assessment. In: J Schneider (ed). Seismic hazard methodology for the Central and Eastern United States. The earthquakes of stable continental regions. Vol. 1. Assessment of large earthquake potential. Palo Alto, CA, USA: EPRI;1994. NP-4726, pp 5-27.

4. Johnston AC. Seismotectonic interpretations and conclusions from the stable continental region seismicity database. In: J Schneider (ed). Seismic hazard methodology for the Central and Eastern United States. The earthquakes of stable continental regions. Vol. 1. Assessment of large earthquake potential. Palo Alto, CA, USA: 
EPRI;1994. NP-4726, pp 4-103.

5. Page R. Aftershocks and microaftershocks. Bull Seism Soc Am 1968;58:1131-68.

6. Cosentino P, Ficara V, Luzio D. Truncated exponential frequencymagnitude relationship in the earthquake statistics. Bull Seism Am 1977;67:1615-23.

7. Mood AM, Graybill F, Boes DC. Introduction to the theory of statistics. 3rd ed. Auckland: McGraw-Hill; 1974.

8. Kendall MG, Stuart A. The advanced theory of statistics. Vol. 2. Inference and relationship. 2nd ed. London: Charles Griffin and $\mathrm{Co.}$ Ltd; 1967.

9. LeCam L. On the assumptions used to prove asymptotic normality of maximum likelihood estimates. Ann Statist 1970;41:802-28.

10. Eadie WT, Drijard D, James FE, et al. Statistical methods in experimental physics. Amsterdam: North-Holland; 1971, Section 8.3.3, p 158.

11. Bain LJ, Engelhardt B. Introduction to probability and mathematical statistics. 2nd ed. Belmont: Duxbury Press; 1992.

12. Petersen MD, Frankel AD, Harmsen SC, et al. Documentation for the 2008 Update of the United States National Seismic Hazard Maps: US Geological Survey Open-File Report 2008-1128, 61. US Department of the Interior, US Geological Survey, 2008.

13. Little RJA, Rubin DB. Statistical analysis with missing data. 1st ed. New York: Wiley; 1987.

14. Tate RF. Unbiased estimation: function of location and scale parameters. Ann Math Statist 1959;30:331-66.

15. Pisarenko VF. Statistical evaluation of maximum possible magnitude, Izvestiya. Earth Physics 1991;27:757-63.

16. Pisarenko VF, Lyubushin AA, Lysenko VB, Golubieva TV. Statistical estimation of seismic hazard parameters: maximum possible magnitude and related parameters. Bull Seism Soc Am 1996;86:691-700.

17. Cooke P. Statistical inference for bounds of random variables. Biometrika 1979;66:367-74.

18. Kijko A. Estimation of the maximum earthquake magnitude, mmax. Pure Appl Geophys 2004;161:1655-81.

19. Kijko A, Graham G. Parametric-historic procedure for probabilistic seismic hazard analysis. Part I: Assessment of maximum regional magnitude mmax. 1998;152:413-42. 